\title{
Match Graph Construction for Large Image Databases
}

\author{
Kwang In Kim ${ }^{1}$, James Tompkin ${ }^{1,2,3}$, \\ Martin Theobald ${ }^{1}$, Jan Kautz ${ }^{2}$, and Christian Theobalt ${ }^{1}$ \\ 1 Max-Planck-Institut für Informatik, Campus E1 4, 66123 Saarbrücken, Germany \\ ${ }^{2}$ University College London, Malet Place, WC1E 6BT London, UK \\ 3 Intel Visual Computing Institute, Campus E2 1, 66123 Saarbrücken, Germany
}

\begin{abstract}
How best to efficiently establish correspondence among a large set of images or video frames is an interesting unanswered question. For large databases, the high computational cost of performing pair-wise image matching is a major problem. However, for many applications, images are inherently sparsely connected, and so current techniques try to correctly estimate small potentially matching subsets of databases upon which to perform expensive pair-wise matching. Our contribution is to pose the identification of potential matches as a link prediction problem in an image correspondence graph, and to propose an effective algorithm to solve this problem. Our algorithm facilitates incremental image matching: initially, the match graph is very sparse, but it becomes dense as we alternate between link prediction and verification. We demonstrate the effectiveness of our algorithm by comparing it with several existing alternatives on large-scale databases. Our resulting match graph is useful for many different applications. As an example, we show the benefits of our graph construction method to a label propagation application which propagates user-provided sparse object labels to other instances of that object in large image collections.
\end{abstract}

Keywords: Image matching, graph construction, link prediction.

\section{Introduction}

The widespread use of digital still and video cameras and the success of social Web services such as Facebook, Flickr, and YouTube has resulted in an enormous amount of publicly available image and video data. Organizing and browsing such large-scale visual data $(100,000+$ images $)$ is more than ever a pertinent problem in computer vision and data mining research. While there have been remarkable developments in the last ten years [1-6], structuring large-scale visual data is still an active research area and any improvements would have wide applicability.

The first step required to organize, annotate, and retrieve from large image and video collections is to establish correspondences among individual images and video frames. This is not only an interesting problem by itself [3], but is also a key component to many potential applications including 3D reconstruction [1, 7, 8], graphical navigation [3, 2], and content-based retrieval [9]. Establishing image correspondence in unconstrained databases is very challenging because of significant variations in the appearances of objects. We focus on the specific case where images are connected by static objects appearing therein, e.g., landmarks and buildings, and this is assumed in most previous

A. Fitzgibbon et al. (Eds.): ECCV 2012, Part I, LNCS 7572, pp. 272-285, 2012.

(c) Springer-Verlag Berlin Heidelberg 2012 
work [1, 7, 3, 2]. This allows us to exploit well-established 3D geometry-based image matching techniques [10]. However, our graph construction technique is general and does not depend on any particular matching technique or data content.

A significant problem with 3D geometry-based matching techniques (as well as other accurate image matching techniques) is that they are computationally expensive and cannot feasibly be applied brute force to large-scale data. Fortunately, for the applications we foresee, our data are inherently sparsely connected. Typically, an image matches only a small subset of the entire database as, for example, photos of city landmarks are naturally geographically separated. We take advantage of this sparsity to quickly estimate a small subset of potential matches and then only verify these with an expensive matching procedure. This saves a significant amount of time versus performing an exhaustive pair-wise matching for a large-scale graph. Further, for many applications, it is useful that our iterative approach produces intermediate results (i.e., only a subset of the entire set of potential matches are identified), allowing services to be provided before computation is complete. Our algorithm is especially useful in this case since it maximizes the number of verified matches given a limited amount of time and computational resources.

Our main contribution is an algorithm which predicts the existence of links among a large set of potential matching candidates. We regard the process of matching as a graph construction where an edge between two nodes (a pair of images) indicates a successful image match. Our system incrementally constructs a graph by iterating between the estimation of potential links and their verification. In our experiments, we demonstrate that our algorithm well-predicts the existence of links and so enables very efficient use of computational resources in the matching stage. This avoids the tremendous task of matching all pairs of images a priori. We also demonstrate that our approach outperforms existing methods on two real-world databases.

Finally, we show how our graph construction can benefit an example application. Here, we choose label propagation: The labels (or annotations) are provided by users for some objects in some images. These labels are typically extremely sparse: as extensive object labeling across an image or video collection is often infeasible, a user may label an object (e.g., 'London Eye') in only a few keyframes in one video. Our algorithm automatically propagates sparse user-provided label data to large unstructured image and video collections. The resulting algorithm enables users to easily share labels between visual data, i.e., to automatically assign labels to as many objects occurrences as possible. Our label propagation framework also includes (semi-automatic) error correction, active label acquisition, and image-based query processing.

\section{Related Work}

Our proposed graph construction algorithm is influenced by many techniques from different disciplines. As such, in the first part of this section we focus only on reviewing work related to organizing images, and we leave a review of data mining research for Sec. 3.2. The second part of this section focuses on reviewing related label propagation algorithms, as we use this application as an example of where our graph construction can benefit. 
Graph Construction for Images. Many aspects of image and video organization are based on a graph structure which encodes the match relations among a given set of images [3, 2, 7, 11]. While there are various choices of algorithm for matching pairs of images, 3D geometry-based matching is particularly relevant when a high level of accuracy is required. To deal with the high computational complexity of 3D geometrybased matching techniques, existing works typically adopt a pre-processing stage to quickly identify a small set of candidates which are later refined with geometry-based matching [3, 11, 7]. For large-scale image collections depicting heterogeneous regions, this approach by itself may not be sufficient as it sacrifices precision to guarantee recall.

Recently, authors have proposed incremental graph construction [3, 7]: First, a sparse graph (i.e., few edges) is built. Second, by exploiting the context of relevant matches, the graph is made dense. By predicting potential edges, a certain measure of graph connectivity (e.g., algebraic graph connectivity [3]) can potentially be maximized.

Our framework improves upon existing incremental graph construction algorithms by focusing directly on increasing the graph connectivity for choosing potential candidates (i.e., to predict edges which are likely to be actual edges). In addition to experimental comparison, we provide a detailed discussion comparing Agarwal et al.'s algorithm [7], Heath et al.'s algorithm [3] and our algorithm in Sec. 3]

Content-Based Retrieval and Annotation as Applications. Closely related to our algorithm, especially to our label propagation application, are previous methods for retrieving and annotating geographic locations (or spatial landmarks). For instance, Kennedy and Naaman [6] used visual features, user tags, and other metadata for clustering and annotating photographs. They exploit metadata and user tags to quickly generate a set of candidate image matches and then refine the results using visual features. Based on cluster coherence and connectivity, representative photographs are identified and are presented as a summary for a location [12]. Zheng et al. [5] proposed a Web-scale landmark recognition engine. The system automatically discovers landmarks by exploiting GPS-tagged photographs and travel guide articles. Then, a large image database is clustered into potential landmarks based on local feature point matching.

Gammeter et al. [4] further developed this idea such that Web-scale annotation is automatically performed at the level of individual objects appearing within images. First, they automatically identify important objects and cluster images based on geo-tagged photos obtained from Flickr. Then, each query image is matched against each cluster, and object bounding boxes are found through connectivity analysis. Other related work in image retrieval and geo-annotation can be found in [13-16].

One important difference between existing content-based annotation work and our label propagation algorithm is that our objective is not to pre-cluster images or to identify important objects (i.e., landmarks) - this is not the goal of label propagation and such services can be built on top of our graph structure. We believe the primary goal of label propagation is to correctly connect as many images as possible such that any label on any object can be propagated, not just the most popular ones.

Video Google [9] was one of the first systems to enabled retrieval of video data. This system quickly identifies regions of interest, each adapted based on the local contexts of images, such that the resulting feature descriptors represent objects in a viewpoint 
invariant way. Our approach is complementary to these 'retrieval'-based approaches since our goal is to connect all pairs of images which contain the same objects. In principle, Video Google could be adopted as a component of our algorithm to quickly generate candidates for more costly 3D geometry-based image matching (cf. Sec. 3).

\section{Our Graph Construction Algorithm}

Our system incrementally constructs a graph by iterating between the estimation of potential links and their verification. To help in this task when connecting large databases of images, our algorithm predicts the existence of links among a large set of potential matching candidates. The remainder of section explains the steps necessary to build the graph and discuss their general properties. While our graph construction algorithm is independent of any specific image matching technique, we exemplify it with 3D geometry-based matching at the end of this section. These techniques will subsequently be used in our label propagation application.

We begin with definitions: For a given image database $\mathcal{I}=\left\{I_{1}, \ldots, I_{l}\right\}$, two images $I_{i}$ and $I_{j}$ are linked if there is an established correspondence. This can be naturally represented as a match graph $\mathcal{G}$ where nodes and edges represent images and links respectively. We refer to the process of identifying links as matching or verification.

Naively pair-wise matching all images may be computationally prohibitive even on medium size databases $\left(10^{6}\right.$ images $)$ when we require computationally expensive match verification. Introducing a filtering phase (as in [7]) may still not be sufficient for very large databases as the number of candidate images to be verified afterward will be prohibitively large. To ease this problem, we propose a two-phase graph construction:

1) Filtering phase For each image, we quickly generates a relatively small set of candidate images upon which to invoke expensive matching. Typically, this phase relies on the vector space structure of image features.

2) Incremental graph construction phase We approximate the final match graph $\mathcal{G}$ with a very sparse graph $\mathcal{G}_{0}$ (i.e., \# edges in $\mathcal{G}_{0} \ll \#$ edges in $\mathcal{G}$ ) which we then incrementally densify. $\mathcal{G}_{0}$ could be obtained either by randomly verifying a small number of image pairs or by relying on domain knowledge (e.g., geo-tagging metadata). Given $\mathcal{G}_{0}$, we iterate the prediction of potential links and their verification:

Prediction: At each step $t$, for each unverified link, we estimate the confidence of that potential link being a real link with the measure in Sec. 3.1

Verification: The first $m$ candidates corresponding to the $m$ highest confidence values are verified and $\mathcal{G}_{t}$ is updated before the $(t+1)$-th step.

We can run a random selection process in parallel to guarantee that all potential links eventually undergo verification (as $t \rightarrow \infty$ ) when using a sub-100\%-accurate confidence measure (for instance, with disconnected subgraphs, see Sec. 5].

\subsection{Link Confidence Measure}

To predict good potential links at step $t$, we need a measure of confidence that a potential link is a real link. For this, we exploit the global connectivity of $\mathcal{G}_{t}$. Throughout 


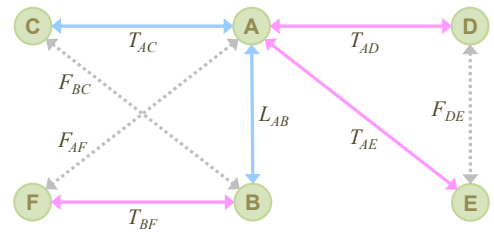

Fig. 1. A schematic diagram of regularization based on similarity relation: the solid and dashed lines indicate verified and unverified links, respectively, while the blue and red lines show positive and negative entries, respectively

iterative graph construction, we maintain a matrix $T$ which contains accumulated link verification results between all pairs of images. A positive entry in $T$ implies that the image pair is similar (e.g., that they contain common feature points). A negative entry in $T$ indicates that the image pair has been verified but was dissimilar (i.e., failed match). A null entry in $T$ shows that no information has been gained so far for that image pair.

A graph Laplacian $L$ is built with the similarity information and is used to regularize the link structure of $\mathcal{G}_{t}$ (both similarity and dissimilarity; $T$ ) based on the paths connected by a similarity relation. We do not use the verified dissimilarity (i.e., the negative entries of $T$ ) in $L$ since dissimilarity is not transitive (the intuitive notion of transitivity, rather than the mathematical one) 1

Figure 1 explains this with examples. Suppose we have six images $A$ to $F$ :

Example 1: If we know that $(A$ and $B)$ and $(A$ and $C)$ are similar, we expect ( $B$ and $C)$ to be similar. However, if $(A$ and $D)$ and $(A$ and $E)$ are dissimilar, then this tells us little about the (dis-)similarity of ( $D$ and $E$ ). In fact, $D$ and $E$ could be identical.

Example 2: We expect that, for images $A, B$, and $F$, the similarity of $(A$ and $B)$ and the dissimilarity of $(B$ and $F)$ suggests the dissimilarity of $(A$ and $F)$.

These examples indicate that both similarity and dissimilarity should be regularized through an edge connected by a similarity relation. That is, the differences of the variables $T_{A C}$ and $T_{B C}$ should be penalized if they are connected by an edge joining $A$ and $B$ and existing in $\mathcal{G}_{t}$.

Formally, for our confidence measure, we use the minimizer of the cost functional:

$$
\mathcal{O}(F)=\frac{1}{2}\left(\lambda \operatorname{tr}\left[F^{\top} L F\right]+\frac{1}{l}\|F-T\|_{\mathcal{F}}^{2}\right),
$$

where $\lambda$ is a regularization parameter, $F(l \times l)$ is the variable matrix whose estimated entries will provide confidences, $\|\cdot\|_{\mathcal{F}}$ is the Frobenius norm, $\operatorname{tr}[\cdot]$ computes the trace, and $L(l \times l)$ is the graph Laplacian constructed from the similarity matrix $W$ :

$$
W_{i j}= \begin{cases}T_{i j} & \text { if } T_{i j}>0 \\ 0 & \text { otherwise. }\end{cases}
$$

The matrix $T$ contains accumulated link verification results up to $(t-1)$ :

$$
T_{i j}= \begin{cases}k\left(I_{i}, I_{j}\right) & \text { if } k\left(I_{i}, I_{j}\right)>0 \\ -1 & \text { if } k\left(I_{i}, I_{j}\right)=0 \\ 0 & \text { if }\left(I_{i}, I_{j}\right) \text { has not been verified. }\end{cases}
$$

\footnotetext{
${ }^{1}$ This transitivity may often fail in practice. Accordingly, we use this notion only for regularizing the labeling process on the graph based on the graph Laplacian.
} 
where $k(I, J)$ encodes the results of verification for a pair $(I, J) . k(\cdot, \cdot)$ is 0 when verification fails and is positive otherwise: If verification of $(I, J)$ produces a strength or score, then $k(I, J)$ is assigned with that specific value. Otherwise, 1 is assigned, indicating successful verification. Section 3.3 shows an example of $k(\cdot, \cdot)$. If the candidate pair $\left(I_{i}, I_{j}\right)$ do not pass through the filtering phase, we assign -1 to $T_{i j}$. The minimizer of Eq. 1 can be easily found by solving a set of linear equations:

$$
(\lambda l L+I) F=T .
$$

We obtain the final confidences by symmetrizing the results (i.e., $F \leftarrow\left(F+F^{\top}\right) / 2$ ).

\subsection{Discussion}

Image Webs. The most related algorithm to our proposed incremental construction method is Image Webs [3]. Similar to our algorithm, Image Webs builds a graph Laplacian and assigns a corresponding score to each unverified edge. This score is used to generate link candidates. Importantly, the Image Webs edge-ordering criterion is not the likelihood of being a potential link, which is in explicit contrast with our algorithm: In Image Webs, the objective of link prediction is to maximize algebraic connectivity. Specifically, the score for an edge is calculated from the difference between corresponding entries of the Fiedler vector for the nodes it joins. Since the Fiedler vector represents a continuous approximation of the discrete cluster indices in spectral clustering, a large difference in Fiedler vector entries indicates the possibility of entries being contained in different clusters. As such, these edges are least supported by the context and are unlikely to be real edges. This observation is further supported by our experiments (see Sec. (4). While algebraic connectivity may be useful in analyzing the global structure of the match graph [3], we believe it not desirable for the intuitive objective of establishing as many relevant connections as possible between images.

Incremental Graph Construction Phase as Link Prediction. Our problem can be regarded as a special instance of the more general link prediction problem where one predicts existences and corresponding properties of links among a set of nodes. This is one of the main problems in data mining research, especially in the context of web connectivity analysis. The use of the graph Laplacian and related methods including diffusion kernels, local random walks, etc 2 have already been demonstrated to be very effective in this context [17-20]. Our algorithm is different from many of these algorithms because our formulation directly uses the links as variables rather than indirectly classifying (or clustering) nodes and inferring the properties of links based on the homogeneity of their joined nodes (Eq. 1). In this way, we can systematically exploit dissimilarity as well as similarity information.

From this perspective, a closely related algorithm is proposed by Kunegis et al. [19]. They construct a signed Laplacian matrix which includes both similarity (positive entries) and dissimilarity (negative entries) information:

$$
\tilde{L}=\tilde{D}-A,
$$

\footnotetext{
${ }^{2}$ As $l \rightarrow \infty$, the graph Laplacian converges to the Laplacian-Beltrami operator which is the generator of the diffusion process on a manifold.
} 
where $A$ is the signed similarity matrix (which is equivalent to $T$ in Eq. 1) which includes both positive and negative entries and $D$ is the diagonal matrix given by:

$$
\tilde{D}_{i i}=\sum_{j}\left|T_{i j}\right| .
$$

Using this matrix, a positive definite kernel matrix $K$ can be constructed:

$$
K=\left(I+\lambda_{L} \tilde{L}\right)^{-1}
$$

where $\lambda_{L}$ is the regularization parameter such that $K_{i j}$ represents the similarity of the $i$-th and $j$-th nodes. This can be used in predicting the existence of potential links: the large value of $K_{i j}$ suggests the existence of a link between the $i$-th and $j$-th nodes, similarly to the matrix $F$ produced by our algorithm. The authors have demonstrated that this algorithm is superior to several existing graph Laplacian-based link prediction algorithms [19]. In the experiments (Sec.44), we demonstrate that the performance of our algorithm is superior to their signed graph Laplacian-based algorithm. Our algorithm is generic and can also be applied to general link prediction problems.

The objective (Eq. 1) is sufficient for the classification setting, i.e., to classify each link into one of two classes of 'similar' and 'dissimilar'. For a general regression setting (which we do not pursue in this paper), one has to modify the objective such that the tentative assignments of properties (assigned to ' 0 ') to unexamined links does not affect the training error:

$$
\mathcal{O}(F)=\frac{1}{2}\left(\lambda \operatorname{tr}\left[F^{\top} L F\right]+\frac{1}{l}\left\|\mathbb{I}^{\prime} \cdot *(F-T)\right\|_{\mathcal{F}}^{2}\right),
$$

where $\mathbb{I}^{\prime}$ is a matrix with $\mathbb{I}_{i j}^{\prime}=1$ if the pair $\left(I_{i}, I_{j}\right)$ is labeled and zero otherwise; and .* represents an element-wise multiplication.

Application Specific Densification. While our criterion for densifying the graph is objective and measurable, it may have to be modified to specifically fit other applications. For instance, in some cases, it might be more desirable to connect a pair of nodes which have lower connectivity rather than nodes which already are strongly connected [3]. We present such a modified algorithm for this case in the supplementary material 3

Exploiting Sparsity. Our algorithm can take advantage of sparsity, especially in constructing the graph Laplacian $L$. When the match graph is disconnected due to sparsity either inherent in the database or caused by a lack of verified links, individual components can be considered separately. In this case, the negative entries of $T$ connecting different partitions can be neglected: within a component, the results obtained by solving Eq. 4 are identical to those obtained by solving the corresponding system on the entire graph. However, in practice, instead of solving each sub-system, we randomly select one connected sub-graph and make predictions only within this graph.

\footnotetext{
${ }^{3}$ We do not evaluate this algorithm since it is difficult to construct an objective criteria.
} 
One computational benefit of Eq. 4] is that its solution can be computed completely independently for each column of $T$. As such, for large-scale partition cases, this can be performed in multi-processor and multi-memory environments.

Error Identification. The function $k(\cdot, \cdot)$ in Eq. 3 may be erroneous in general. For instance, 3D geometry-based matching (see Sec. 3.3 may fail, and so there are cases where an incorrect link is mistakenly supported by a non-negligible number of feature correspondences. The predicted link confidences can also be used to identify potential matching mistakes that can subsequently be examined by human operators.

We use the confidence measure (Eq.10 to re-assign the weights of existing links, i.e., to replace $k\left(I_{i}, I_{j}\right)$ with $F_{i j}$. The links with the largest decrease in link weight become candidates for verification. Our preliminary experiments suggested that the best use of this approach $i s$ to enumerate the nodes which have degree higher than a threshold $T_{d}$ (which is fixed at 5) and to verify each such node in the first several candidates. The details of this experimental setting are described in Sec.4.

The graph construction step parameters are optimized by performing cross-validation on a small data set. Table 1 summarizes the optimized parameters. See supplementary material for more options for error correction.

\subsection{Graph Construction with 3D Geometry-Based Matching}

While our graph construction technique can be used with any type of image matching algorithm, to demonstrate our approach we implement our two-phase graph construction specifically with 3D geometry-based matching, as follows:

1) Filtering phase Each frame is represented with a histogram of a bag of words [21]. For each image, the corresponding candidates are the images with distances smaller than a given threshold $T_{F}$. We use the spatial pyramid matching kernel [22] as an inverse distance measure.

2) Incremental graph construction phase To match image pairs, we extract SIFT features [21] from each image. Then, for a given pair of images, RANSAC estimates feature correspondences that are most consistent according to the fundamental matrix, similar to other related methods [10, 3, 2].

In Eq. 3] $k(\cdot, \cdot) \in[0,1]$ is the normalized feature correspondence measure given as:

$$
k\left(I_{i}, I_{j}\right)=\frac{2\left|\mathcal{M}\left(I_{i}, I_{j}\right)\right|}{\left|\mathcal{S}\left(I_{i}\right)\right|+\left|\mathcal{S}\left(I_{j}\right)\right|},
$$

where $\mathcal{S}(I)$ and $\mathcal{M}(I, J)$ are the set of features (SIFT descriptors) calculated from image $I$ and the set of features matches for images $I$ and $J$, respectively. To ensure that the numbers of SIFT descriptors extracted from any pair of images is comparable, all images are scaled to identical heights (540 pixels) 4 Intuitively, $k(I, J)$ is close to 1 when two images $I$ and $J$ contain common features and are similar.

\footnotetext{
${ }^{4}$ Even though the SIFT detectors are theoretically scale invariant, the corresponding implementation is typically scale-dependent. It is common to limit the lowest scale of local extrema in SIFT detection to make the algorithm robust against noise.
} 
Table 1. Parameters for experiments

\begin{tabular}{cccc}
\hline$T_{F}$ & $T_{d}$ & $\lambda$ & $\lambda$ (error identification) \\
\hline 1.5 & 5.0 & $1 \cdot 10^{-1}$ & $0.5 \cdot 10^{-3}$ \\
\hline
\end{tabular}

\subsection{Implementation Details}

In feature point matching, we set the link weight $k\left(I_{i}, I_{j}\right)$ to be zero (i.e., the link is removed) if the number of feature point correspondences between $I_{i}$ and $I_{j}$ is smaller than 12. The regularization parameter $\lambda$ in Eq. 11 is determined based on cross-validation. For this, 2, 000 images are randomly selected from the data set A (see Sec. 4) and feature matching is performed for every pair of images which pass through the filtering phase.

To construct $T$ and $L$, we randomly selected $5 \%$ of the established links plus $20 \%$ of the image pairs with verified non-existence links. For each value of $\lambda$, we construct 10 sets of these experimental databases, in each of which 100 candidates with the highest confidence values $(F)$ are compared with the link examination results. The number of established links is counted among them. $\lambda$ is then determined as the maximizer of the sum of correct links. In general, the above-mentioned 'link examination results' may contain some errors (see Fig. 3 of the supplementary material for an example). However, these errors very rarely occur and do not critically bias the selection of $\lambda$.

The $\lambda$ for the error correction process is optimized similarly. For each randomly selected node, we add an incorrect link and estimate the confidences of all links. In the result, we count the occurrences of the case where the first candidate correctly identifies the incorrect link.

The threshold $T_{F}$ for the filtering phase is set at 1.5. This value was decided as the maximum value which did not result in the removal of any correct links in an experiment with a small dataset of 300 images. On our database (set B; see Sec. (4), this value resulted in reducing approximately $70 \%$ of candidates.

\section{Experiments}

We have tested our system on two databases consisting of 118,000 and 102,361 images. In the first database (set A), the images were downloaded from Flickr by searching for 35 keywords of locations in London. In the second database (set B), the images are obtained as frames selected from 249 videos, each up to 20 minutes in length, taken at and between several locations in London. These videos include landmarks such as Big Ben, the London Eye, and St Paul's Cathedral. The footage also includes general street footage between each landmark. The videos vary in location, time, foreground objects, viewpoint, and camera model. In the following sections, first we evaluate the performance of our main contribution of our graph construction algorithm. Then, we provide an example application scenario of label propagation.

\subsection{Evaluation of Incremental Graph Construction Performance}

We performed matching on around $10^{7}$ randomly selected candidate pairs which had passed through the filtering phase. This resulted in approximately 400 non-trivial (i.e., 
size larger than 1) subgraphs for set A, and 2000 for set B. Set B has a much higher number of non-trivial subgraphs since the individual images are sampled densely from videos and so it has high connectivity. Many of the images in set A do not correspond to any spatial landmarks (e.g., someone's face) and are not connected to any other images, so set A is very sparse. The average size of non-trivial subgraphs was around 4 (set A) and 15 (set B) while some subgraphs were several thousand large.

For a randomly selected connected sub-graph, 30 potential links with high confidence values were verified and the graph was updated (see Sec.3). During sub-graph selection, we excluded those sub-graphs which have less than 20 nodes. This process was repeated 50 times and the average hit ratio is examined. The hit-ratio is defined as the number of successfully verified links divided by the number of total verified links. For comparison, we have implemented two existing algorithms for graph construction for images. Our adaptation of the graph augmentation in Building Rome in a Day [7] uses a heuristic based on the transitivity characteristic of the similarity discussed in Sec. 3, if an image $I_{i}$ links to another image $I_{j}$, and $I_{j}$ is connected to $I_{k}$, then the pair $\left(I_{i}, I_{k}\right)$ are verified. Image Webs [3] extracts the Fiedler vector of the graph Laplacian $L$ and assigns confidence based on the pairwise differences of elements of the Fiedler vector (see Sec.3). We also compare with an established method for general link prediction: the signed graph Laplacian [19] (see Sec.3.2 for discussion). Table 2 summarizes these results.

Our proposed method was tested with two settings. In the first setting (denoted 'sim. only'), only the positive entries of link verification are used. In the second setting, both the positive and negative results are used. The results indicate that our method is significantly better in terms of hit ratio than the heuristic of [7], than using the Fiedler vector [3] or than using the signed graph Laplacian [19]. The improvement obtained by using negative (dissimilarity) labels over the case of using positive labels is also noticeable even though it is not as dramatic an improvement. Furthermore, even though images are randomly sampled, in repeated experiments using negative examples produced a constant hit ratio improvement over using only positive examples.

For all experiments, the number of examined candidate links for calculating the hitratio is the same for all algorithms: For each state of evaluation, the graph is prepared with the same setting and with exactly the same number of predictions made. To make fair comparisons with this graph, Image Webs [3], the signed graph Laplacian [19], and Building Rome in a Day [7] are not their original versions but are our own implementations as they use the filtering stage of our algorithm (however, our implementations of the link prediction parts of these techniques are as written in their respective papers). Accordingly, the hit ratio reported Table 2 reflects only the link prediction performance of each algorithm. From an application perspective, a better performance criteria than the hit-ratio would be the number of found edges normalized by the number of total (potential) links in the graph - the absolute performance. However, this requires matching every possible pair of images in the graph, which is computationally infeasible for realistically large data sets such as our example data sets.

The time complexity of our algorithm is $O\left(l^{3}\right)$. However, as discussed in Sec. 3.2, our algorithm takes advantage of sparsity and disconnectedness of the graph. Accordingly, the time complexity reduces to $O\left(p^{3}\right)$, where $p$ is the size of the largest connected subgraph. As such, this theoretical complexity of $O\left(l^{3}\right)$ almost never occurs in practice. 
Table 2. Hit ratios of different link prediction algorithms

\begin{tabular}{lcc}
\hline \multirow{2}{*}{ Algorithm } & \multicolumn{2}{c}{ Hit ratio } \\
\cline { 2 - 3 } & \multicolumn{2}{c}{ Set A Set B } \\
\hline Image Webs [3] & 0.25 & 0.12 \\
Random selection & 0.32 & 0.17 \\
Signed graph Laplacian [19] & 0.35 & 0.46 \\
Building Rome in a Day [7] & 0.49 & 0.82 \\
Proposed method (sim. only) & 0.58 & 0.94 \\
Proposed method & 0.60 & 0.97 \\
\hline
\end{tabular}

Table 3. Hit ratios of the first 3 candidates for link error identification

\begin{tabular}{lccc}
\hline Candidates & 1st & 2nd & 3rd \\
\hline Cumulative hit ratio & 0.94 & 0.99 & 1.00 \\
\hline
\end{tabular}

In experiments, we have almost linear complexity since the individual sub-graphs are sparse. Even for the largest sub-graph (order of thousands), our prediction algorithm took on average 0.78 second The times taken for Image Webs and Building Rome in a Day were 0.13 and 0.03 second, respectively. The prediction time of our algorithm is longer than that of other algorithms; however, since currently the main bottleneck of these algorithms is the matching stage, this is not a problem. Given that, our whole algorithm achieves higher connectivity than other algorithms for a given fixed amount of computation time.

We have also evaluated the performance of our link error identification. Since link error is very rare, it is hard to evaluate performance systematically. To facilitate evaluation, we prepared a small dataset of 3,000 images from set B and built a ground truth link verification set by exhaustively performing pairwise matching. Then, for each randomly selected 500 nodes, we randomly added an 'incorrect' link and computed the hit-ratio within the first 3 candidates suggested by our algorithm. Numbers of candidates ranged from 5 (the minimum degree which invokes the error identification process) to hundreds, while the average number of candidates is 18.51 . Table 3 summarizes the results showing that we can very accurately identify erroneous links.

\subsection{Application: Label Propagation in Images and Videos}

In this section, we show the example application of label propagation (LP). This application requires a graph structure to be constructed from an image database, and here our algorithm provides this structure.

Our supplementary material contains additional information on 1) active label acquisition and how we can provide label suggestions, 2) how new images can be added to existing graphs in cases where the database is not fixed a priori, and 3) additional steps required for processing videos instead of images. 
Labels. A provided label for an object of interest consists of a bounding box of the object observed in an image and a corresponding tag. A tag can be an objective name of a spatial landmark (e.g., 'Big Ben'), a subjective description, or low-level machine interpretable code (e.g., a GPS log, an index of a database entry, a link to a Wikipedia entry or Google search result, etc.). In general, there can be multiple labels attached to a single object. The result of LP on an image database $\mathcal{I}=\left\{I_{1}, \ldots, I_{l}\right\}$ is represented as a list of images in $\mathcal{I}$ containing the object, the corresponding bounding boxes, and the tags. We refer to this result as the propagated labels.

Label propagation can be performed immediately after the match graph $\mathcal{G}$ is constructed 5 Each time a label is generated for an image, it is immediately transferred to its neighbors in $\mathcal{G}$. Those propagated labels are again transferred to their neighbors. In this way, the depth of a label increases. This depth is defined to be zero for a userprovided label. This potentially cyclical procedure can generate infinite instances of the same labels (i.e., the same tag with potentially different bounding boxes) occurring even in a single image. We resolve this by selecting and retaining only one bounding box per tag with the minimum depth among them.

Transferring a label from one image to another uses feature point correspondences from 3D scene geometry estimation. For each feature point within the label bounding box, the corresponding feature is retrieved in the target image (if it exists). The propagated label is obtained as the minimum bounding box containing all retrieved feature points (Fig. 2a). In general, the result of this procedure is very conservative: the bounding box may shrink as label depth increases (Fig. 2b). This can be attributed either to the sparseness of feature point matches (Fig. 2a) or to the sparseness of the matching graph (Fig. 2b). While we retain all bounding box information, in the user interface, the labels are displayed at the center of the bounding box (Fig. 3). This avoids the rather difficult problem of object segmentation in images. For many LP applications that we foresee, the location of a part of an object is already sufficient.

During label transfer, the influence of erroneous feature matches is limited by removing outlier correspondences: if the $x$ and $y$ coordinate values of a displacement vector corresponding to a point correspondence are larger than 4 times the corresponding standard deviations computed on all correspondences, the correspondence is removed. In preliminary experiments, this completely removed label transfer mistakes. However, LP was slightly more conservative - we removed some correct feature correspondences.

\section{Discussion}

Our algorithm does not support connecting two disjoint graphs: Our algorithm uses the graph Laplacian as a regularizer which, for disconnected graphs, does not penalizes any differences in corresponding function assignments for different connected components. Instead, to guarantee that all potential links joining different components of a graph eventually undergo the verification process, we run a random selection process

\footnotetext{
${ }^{5}$ In our application scenario, we provide LP immediately after the construction of the initial match graph $\mathcal{G}_{0}$; we don't have to wait until the incremental graph construction process stabilizes. As such, our system performance will initially be poor, but it will quickly improve as the match graph becomes denser.
} 


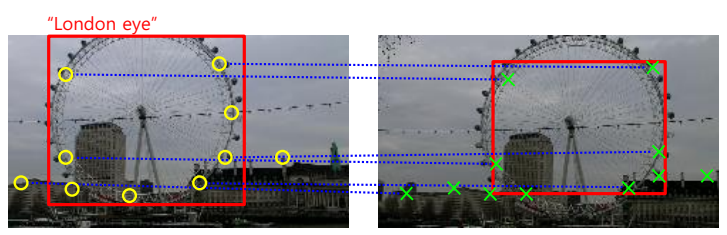

(a)

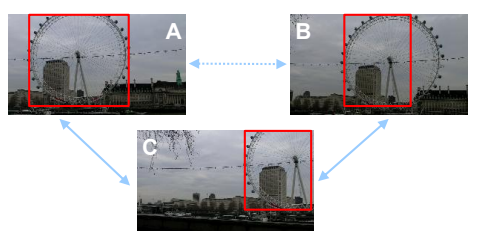

(b)

Fig. 2. A schematic diagram of label transfer: (a) The propagated label (the rectangle in the right image) is obtained as the minimal bounding box of the feature points (green crosses) which have the corresponding matching features contained in the provided label (the rectangle in the left image); (b) Image B label is obtained from the user provided label in A propagated through $\mathrm{C}$. At a certain time step $t$, the link between A and B may yet have been established.

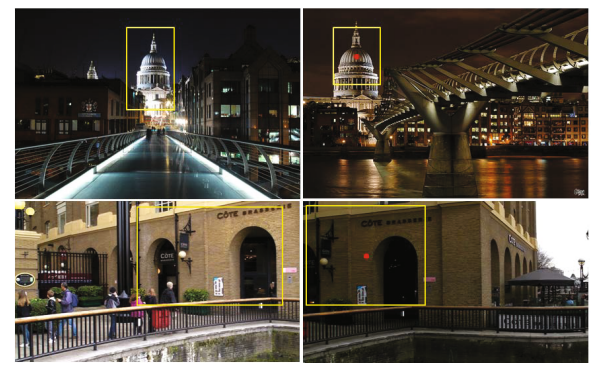

Fig. 3. Examples of label propagation: Left: user-provided labels. Right: propagated labels. Yellow rectangles and red circles show the bounding boxes and the corresponding centers, respectively. The last image shows that the object of interest does not need to correspond to any famous landmark - the tag is a subjective annotation for a brasserie.

in parallel to our estimated confidence-based algorithm. We are not aware of any existing algorithm which is capable of solving this interesting problem. Image Webs shares the same limitation since it is also based on the graph Laplacian: the eigenvectors of a graph Laplacian corresponding to a graph consisting of several connected components are essentially the same as the collection of eigenvectors of individual graph Laplacians (with properly attached zeros to make two different sets of eigenvectors have the same dimension). Future work should explore this direction.

There are other various different directions for future work. In graph construction, matching performance should be significantly improved by exploiting GPS information provided by modern cameras. Alternatives for the current image matching algorithm (RANSAC-based feature point correspondence estimation) should also be explored. Our graph construction algorithm is generic and can be used for non-image data. Additional use cases for the basic algorithm could be explored. Additional discussion on label propagation is provided in the accompanying supplementary material.

Conclusion. This paper presents an algorithm for efficient match graph construction on large-scale image and video databases. Our algorithm straightforwardly exploits the intermediate match results and predicts potential matches, which turns out to be superior to several existing algorithms in terms of hit ratio. This enables maximizing the connectivity of the graph for limited computational resources. Our algorithm finds applications in many contemporary problems in large media set processing, e.g., label propagation. 
Acknowledgement. We thank Nils Hasler for proof reading.

\section{References}

1. Snavely, N., Seitz, S.M., Szeliski, R.: Photo tourism: exploring photo collections in 3D. ACM TOG (Proc. SIGGRAPH) 25, 835-846 (2006)

2. Raguram, R., Wu, C., Frahm, J.M., Lazebnik, S.: Modeling and recognition of landmark image collections using iconic scene graphs. IJCV 95(3), 213-239 (2011)

3. Heath, K., Gelfand, N., Ovsjanikov, M., Aanjaneya, M., Guibas, L.J.: Image webs: computing and exploiting connectivity in image collections. In: Proc. IEEE CVPR, pp. 3432-3439 (2010)

4. Gammeter, S., Bossard, L., Quack, T., Gool, L.V.: I know what you did last summer: objectlevel auto-annotation of holiday snaps. In: Proc. ICCV, pp. 614-621 (2009)

5. Zheng, Y.T., Zhao, M., Song, Y., Adam, H., Buddemeier, U., Bissacco, A., Brucher, F., Chua, T.S., Neven, H.: Tour the world: building a web-scale landmark recognition engine. In: Proc. IEEE CVPR, pp. 1085-1092 (2009)

6. Kennedy, L., Naaman, M.: Generating diverse and representative image search results for landmarks. In: Proc. WWW, pp. 297-306 (2008)

7. Agarwal, S., Snavely, N., Simon, I., Seitz, S.M., Szeliski, R.: Building Rome in a day. In: Proc. ICCV (2009)

8. Tompkin, J., Kim, K.I., Kautz, J., Theobalt, C.: Videoscapes: exploring sparse, unstructured video collections. ACM TOG (Proc. SIGGRAPH) 68, 1-12 (2012)

9. Sivic, J., Zisserman, A.: Video Google: A text retrieval approach to object matching in videos. In: Proc. ICCV, pp. 1470-1477 (2003)

10. Hartley, R.I., Zisserman, A.: Multiple View Geometry in Computer Vision, 2nd edn. Cambridge University Press (2004)

11. Philbin, J., Sivic, J., Zisserman, A.: Geometric latent Dirichlet allocation on a matching graph for large-scale image datasets. IJCV 95(2), 138-153 (2011)

12. Weyand, T., Leibe, B.: Discovering favorite views of popular places with iconoid shift. In: Proc. ICCV, pp. 1132-1139 (2011)

13. Hays, J., Efros, A.A.: IM2GPS: estimating geographic information from a single image. In: Proc. IEEE CVPR, pp. 1-8 (2008)

14. Kleban, J., Moxley, E., Xu, J., Manjunath, B.S.: Global annotation on georeferenced photographs. In: Proc. CIVR, pp. 1-8 (2009)

15. Serdyukov, P., Murdock, V., van Zwol, R.: Placing flickr photos on a map. In: Proc. SIGIR, pp. 484-491 (2009)

16. Crandall, D.J., Backstrom, L., Huttenlocher, D., Kleinberg, J.: Mapping the world's photos. In: Proc. WWW, pp. 761-770 (2009)

17. Kunegis, J., Lommatzsch, A.: Learning spectral graph transformations for link prediction. In: Proc. ICML, pp. 561-568 (2009)

18. Backstrom, L., Leskovec, J.: Supervised random walks: predicting and recommending links in social networks. In: Proc. WSDM, pp. 635-644 (2011)

19. Kunegis, J., Schmidt, S., Lommatzsch, A., Lerner, J., Luca, E.W., Albayrak, S.: Spectral analysis of signed graphs for clustering, prediction and visualization. In: Proc. ICDM, pp. 559-570 (2010)

20. Lü, L., Zhou, T.: Link prediction in complex networks: a survey. arXiv:1010.0725v1 (2011)

21. Lowe, D.G.: Distinctive image features from scale-invariant keypoints. IJCV 60(2), 91-110 (2004)

22. Lazebnik, S., Schmid, C., Ponce., J.: Beyond bags of features: spatial pyramid matching for recognizing natural scene categories. In: Proc. IEEE CVPR, pp. 2169-2178 (2006) 